\title{
O Ensino de Habilidades Clínicas e a Aplicabilidade de um Guia Simplificado de Exame Físico na Graduação de Medicina
}

\author{
The Teaching of Clinical Skills and the \\ Applicability of a Simplified Guide for Physical \\ Examination in the Undergraduate Medical \\ Program
}

\author{
PALAVRAS-CHAVE \\ - Exame Físico. \\ - Educação Médica. \\ - Guia. \\ - Educação de Graduação em \\ Medicina.
}

Salomão Georges Kahwage Neto ${ }^{I}$ Tiago Kiyoshi Kitabayashi Bragal Márcia Bitar Portella ${ }^{l}$ Régis Bruni Andriolo

\begin{abstract}
RESUMO
Introdução: Apesar dos avanços tecnológicos, a anamnese e o exame físico permanecem as ferramentas diagnósticas mais importantes e eficazes diante de um caso clínico. No entanto, muitos alunos concluem o curso médico com deficiências nessas habilidades essenciais. A falta de padronização do exame físico é considerada uma das principais dificuldades no ensino-aprendizagem. Objetivo: Avaliar as habilidades clínicas dos estudantes do internato de Medicina e a aplicabilidade de um guia simplificado de exame físico para o aperfeiçoamento dessas habilidades. Métodos: O estudo foi analítico, quantitativo do tipo comparativo antes e depois, realizado com alunos de Medicina em estágio de Clínica Médica no período de janeiro a fevereiro de 2014. Os estudantes foram treinados com o guia simplificado por um período de três semanas. Os alunos tiveram seu exame clínico avaliado em 13 itens: sinais vitais, exame da cavidade oral, fundoscopia, otoscopia, exame da tireoide, exame cardiovascular, pulmonar, abdominal, linfonodos, medidas antropométricas, índice tornozelo-braquial (ITB), exame neurológico, exame das mamas (pacientes mulheres) ou dos testículos (pacientes homens). O resultado da avaliação de cada item foi classificado em três categorias: avaliação completa, avaliação parcial e avaliação ausente. Resultados: Ao todo, participaram 31 estudantes. Observou-se melhora significativa de quase todos os itens em relação à avaliação completa após a capacitação com o guia: sistema cardiovascular $(3,23 \%$ versus $74,19 \%$, antes e depois do treinamento, respectivamente, $p<0,01)$; sistema pulmonar (22,58\% versus $90,32 \%, p<0,01)$; abdome ( $22,58 \%$ versus $74,19 \%, p=0,01)$; sinais vitais $(16,13 \%$ versus $100 \%, p<0,01)$; palpação de linfonodos ( $6,45 \%$ versus $77,42 \%, p<0,01)$; exame neurológico $(0 \%$ versus $22,58 \%, p=0,02)$; palpação da tireoide ( $0 \%$ versus $61,29 \%, p<0,01)$; exame da cavidade oral $(6,45 \%$ versus $67,74 \%, p<0,01)$; medidas antropométricas ( $0 \%$ versus $45,16 \%, p<0,01)$; exame das mamas ( $0 \%$ versus $36,84 \%, p=0,02)$; fundoscopia ( $0 \%$ versus $32,26 \%, p<0,01)$; otoscopia $(0 \%$ versus $64,52 \%, p<0,01$ ); avaliação do indice tornozelo-braquial ( $0 \%$ versus $83,87 \%, p<0,01$ ); exame dos testículos ( $0 \%$ versus $8,33 \%, p=1,0$ ). Foi possivel observar também um aumento de 280,7\% na pontuação mediana do desempenho dos alunos após o treinamento (1,92 versus 7,31 pontos, $P<$ 0,001). Notou-se ausência de correlação significativa entre o desempenho dos alunos e o tempo de permanência no curso $(R 2=0,1242 ; P=0,0515)$. Conclusões: Há um déficit grande no ensino de habilidades clínicas durante a graduação de Medicina. Como uma solução eficaz, um guia simplificado sequencial de exame clínico pode servir no treinamento de estudantes de Medicina.
\end{abstract}




\section{KEYWORDS}

- Physical Examination.

- Medical Education.

- Guide.

- Undergraduate Medical Education.
Recebido em: 10/01/2017

Aprovado em: 13/03/2017

\section{ABSTRACT}

Introduction: In spite of technological advances, anamnesis and physical examination are still the most important and effective early diagnostic tools in any clinical case. However, many students graduate in medicine lacking these essential skills. The absence of a standardized physical examination is one of the main difficulties in the teaching-learning process. Objective: To evaluate the clinical skills of medical intern students and the applicability of a simplified physical examination guide for improving these skills. Methods: This was an analytical and quantitative-approach study, which compared before-and-after information among general medicine student interns from January to February 2014. The students were trained with the simplified guide for a 3-week period and their clinical examination was evaluated in 13 items: vital signs, examination of oral cavity, fundoscopy, otoscopy, thyroid examination, cardiovascular examination, pulmonary examination, abdominal exam, lymph nodes, anthropometric measurements, ankle-brachial index (ABI), neurological examination, examination of the breasts or testicles (examination of breasts for female patients and testicles for male patients). The result of the evaluation of each item was classified into 3 categories: complete evaluation, partial evaluation and no evaluation. Results: A total of 31 students participated. Significant improvement was found in almost all items in relation to the complete evaluation after training with the guide: cardiovascular system (3.23\% versus $74.19 \%$, before and after training, respectively, $p<0.01)$, pulmonary system $(22.58 \%$ versus $90.32 \%, p<0.01)$, abdominal exam $(22.58 \%$ versus $74.19 \%, p=$ $0.01)$, vital signs $(16.13 \%$ versus $100 \%, p<0.01)$, palpation of lymph nodes $(6.45 \%$ versus $77.42 \%$, $p<0.01)$, neurological examination ( $0 \%$ versus $22.58 \%, p=0.02)$, thyroid palpation ( $0 \%$ versus $61.29 \%, p<0.01)$, examination of oral cavity $(6.45 \%$ versus $67.74 \%, p<0.01)$, anthropometric measurements ( $0 \%$ versus $45.16 \%, p<0.01)$, breast examination $(0 \%$ versus $36.84 \%, p=0.02)$, fundoscopy $(0 \%$ versus $32.26 \%, p<0.01)$, evaluation of the ankle-brachial index $(0 \%$ versus $83.87 \%$, $p<0.01$ ), examination of the testicles ( $0 \%$ versus $8.33 \%, p=1.0)$. An increase of $280.7 \%$ was also observed in the students' median score after training (1.92 versus 7.31 points, $P<0.001)$. There was no significant correlation between student performance and time on the medical course ( $R 2=0.1242$; $P=0.0515)$. Conclusions: There is a large deficit in teaching clinical skills during undergraduate medical courses. As an effective solution, a simplified sequential clinical examination guide can serve as training for medical students.

\section{INTRODUÇÃO}

O exame físico é fundamental para orientar o diagnóstico e a terapêutica ${ }^{1}$. No entanto, mesmo os residentes de Clínica Médica demonstram deficiências significativas nas habilidades clínicas, que são consideradas importantes durante o progra$\mathrm{ma}^{2}$. Um estudo realizado por Mavis revelou que os estudantes gastavam apenas $20 \%$ do tempo de que realmente deveriam dispor para treinar as habilidades do exame físico. As evidências comprovaram que o percentual de tempo restante foi utilizado para analisar livros didáticos e apostilas ${ }^{3}$.

Em razão das notícias incontestáveis de identificação dessas deficiências, o ensino do exame físico assume estatísticas cada vez mais deploráveis, justo porque no dia a dia se torna uma opção cada vez mais decrescente. Assim, enquanto, em 1960,75\% do ensino clínico se efetuavam à beira do leito, em
1978 esse percentual caiu para $16 \%{ }^{4}$. Como resultado, o exame físico continua em franca decadência, sobretudo em face de seu desuso entre os alunos e médicos assistentes, o que na prática se traduz por uma redução da familiaridade com os sinais clínicos e da utilidade da semiologia, levando a uma dependência crescente de exames laboratoriais e de imagem ${ }^{5,6}$.

Apesar dos avanços tecnológicos, as habilidades para realizar história, para proceder ao exame físico e para se comunicar com o paciente permanecem as ferramentas diagnósticas mais importantes e eficazes diante de um caso clínico. No entanto, muitos alunos concluem o curso médico com deficiências nessas habilidades essenciais ${ }^{7}$. Tal constatação reforça a necessidade de que, durante a graduação médica, os educadores voltem a sua atenção para a avaliação da competência clínica, caracterizada como um conjunto de conhecimentos, 
habilidades técnicas e de comunicação, empatia, propedêutica e raciocínio clínico ${ }^{8}$. Cuidadosamente integrado com as novas tecnologias, o exame físico continuará a ser fundamental para a prática médica.

Em um ambiente clínico, muitos alunos se sentem frustrados pela dificuldade de recordar conhecimentos adquiridos previamente e por serem incapazes de aplicá-los no caso clínico em questão. Os docentes, por sua vez, ficam surpresos com os alunos, que parecem ter retido tão pouco. O problema talvez esteja na separação entre o aprendizado clínico e o ambiente em que é aplicado9.

Talvez por falta de um guia básico de exame físico com uma sequência simples e prática, os estudantes de Medicina não conseguem aplicar o conhecimento prévio na prática, negligenciando pontos semiológicos importantes. Muitos médicos, por exemplo, não medem a circunferência abdominal em consultas de rotina. Este dado é importante para avaliar o risco cardiovascular de um paciente; em homens com mais de $94 \mathrm{~cm}$ e em mulheres com mais de $80 \mathrm{~cm}$ de circunferência, há um risco aumentado de complicações metabólicas e cardiovasculares ${ }^{10}$. Outro dado clínico também negligenciado é o índice tornozelo-braquial, que permite o diagnóstico da doença arterial periférica e também é um fator prognóstico de eventos de mortalidade cardiovascular ${ }^{11,12}$.

Na prática, não existe um exame físico de rotina com uma sequência padrão para exploração dos diversos sistemas. Existem muitos modelos para a realização do exame físico com vantagens e desvantagens, a maioria com um checklist extenso e complexo. A elaboração de um guia simplificado com uma sequência estruturada do exame físico pode servir como modelo para o estudante de Medicina. É a repetição sistemática que pode tornar o estudante um médico hábil em exame físico.

Portanto, o objetivo deste trabalho foi avaliar as habilidades clínicas dos estudantes do internato de Medicina e a aplicabilidade de um guia simplificado de exame físico para o aperfeiçoamento dessas habilidades.

\section{MÉTODO}

O estudo foi analítico, quantitativo do tipo comparativo antes e depois. O trabalho, após a aprovação do Comitê de Ética em Pesquisa da Universidade do Estado do Pará (CEP-Uepa), foi realizado no Complexo Hospitalar Jean Bitar (CHJB), um dos hospitais de ensino da Uepa, na cidade de Belém. Foram incluídos alunos de ambos os sexos, regularmente matriculados no internato do curso de Medicina da Uepa e que realizaram o módulo de Clínica Médica no hospital, de janeiro a fevereiro de 2014. Os pacientes a serem examinados foram os que estavam internados na enfermaria de Clínica Médica. Todos os participantes, alunos e pacientes examinados, assinaram o Termo de Consentimento Livre e Esclarecido.

A sequência do guia simplificado de exame físico foi estruturada para manter o movimento do examinador a um mínimo e limitar a frequência com que o paciente teria que se levantar, permitindo a exploração de áreas do corpo que façam parte de diferentes sistemas de órgãos. Este é um dos muitos roteiros possíveis que permitem realizar um exame físico completo.

A pesquisa foi realizada em duas etapas: no início do estágio em Clínica Médica sem o treinamento com o guia de exame físico, e no final do estágio, após o treinamento com o guia.

No primeiro momento, os estudantes que haviam acabado de ingressar no estágio de Clínica Médica do internato registraram os dados do exame físico nos prontuários sem qualquer treinamento com o guia. $\mathrm{O}$ aluno foi acompanhado pelo médico residente responsável pelo leito com o objetivo de observar se o exame foi realizado. Depois, foram analisados os dados registrados sobre o exame físico no prontuário de cada paciente por meio de uma ficha de avaliação que analisou quais dos 13 aspectos semiológicos foram examinados pelos estudantes: sinais vitais, exame da cavidade oral, fundoscopia, otoscopia, exame da tireoide, exame cardiovascular, exame pulmonar, exame abdominal, exame dos linfonodos, medidas antropométricas, índice tornozelo-braquial (ITB), exame neurológico, exame das mamas (pacientes mulheres) ou dos testículos (pacientes homens).

A avaliação de cada domínio semiológico foi classificada em "avaliação completa", "avaliação parcial" ou "avaliação ausente".

O registro dos sinais vitais foi considerado completo se a pressão arterial, a temperatura, a frequência do pulso e a frequência respiratória estivessem anotadas. $\mathrm{O}$ exame cardiovascular foi considerado completo se houvesse o registro da ausculta cardíaca, da palpação de pulsos periféricos e do índice tornozelo-braquial. No caso do exame pulmonar, se descritas a ausculta e a palpação ou percussão torácica; no exame abdominal, se descritos os dados da inspeção, percussão, ausculta e palpação do abdome. $\mathrm{O}$ exame neurológico foi considerado completo se nele constasse a descrição da marcha, do exame pupilar, dos movimentos oculares, da força muscular e dos reflexos periféricos. Em relação às medidas antropométricas, se descritos o peso, a altura, o índice de massa corpórea (IMC) e a circunferência abdominal.

O exame dos linfonodos foi considerado completo se houvesse o registro da inspeção e palpação das principais cadeias ganglionares: cabeça e pescoço, axilares, epitrocleares e inguinais. Quando palpáveis, o observador deveria descrever as 
seguintes características: localização, tamanho, consistência, fixação à pele e planos profundos, sensibilidade e alterações da pele (rubor, temperatura, fístulas, cicatrizes). O exame da tireoide, para ser completo, deveria conter as seguintes informações obrigatórias na palpação: volume, consistência, mobilidade, superfície e frêmitos.

Quanto ao exame da cavidade oral, foram disponibilizados uma fonte de luz (lanterna) e abaixadores de língua para sua execução. A descrição completa deveria conter informações sobre os achados da inspeção dos lábios, mucosa bucal, gengivas, dentes, palato, língua e faringe. Para fundoscopia, foi disponibilizado um oftalmoscópio e foi considerada completa se houvesse registro da inspeção dos fundos dos olhos com a descrição do disco óptico, cruzamentos A-V, lesões retinianas e área da mácula. Para a otoscopia, foi disponibilizado um otoscópio com espéculos e foi considerada completa se houvesse o registro dos achados da inspeção do canal auditivo e da membrana timpânica.

O exame das mamas deveria incluir os achados da inspeção e da palpação de cada mama com a paciente deitada. O exame dos testículos deveria incluir os achados da inspeção da bolsa escrotal (contornos e pele) e da palpação de cada testículo (dor, nódulo e consistência). O ITB deveria conter a pressão sistólica (PAS) obtida na perna direita, a pressão sistólica (PAS) obtida no braço direito e o valor da razão PAS perna direita/PAS braço direito.

Estes critérios foram estabelecidos como uma base para avaliação indireta da qualidade do exame efetuado. Os exames que não cumpriram todos os quesitos para serem considerados completos foram classificados como "avaliações parciais". As descrições semiológicas que se expuseram como "sem particularidades" ou "nada digno de nota" foram consideradas incompletas. Também foram atribuídos como ausentes os domínios do exame físico que não tiveram assinalados, de maneira clara, se foram considerados "completos", "parciais" ou "ausentes", isto é, os dados estavam indisponíveis, por mau preenchimento, na ficha de avaliação.

Após o primeiro momento, os estudantes foram treinados com o guia de exame físico simplificado, que tem duração aproximada de 30 minutos. Inicialmente, a sequência foi realizada pelo instrutor e depois pelo aluno, que continuou recebendo treinamento diário do instrutor com a sequência do guia durante o período do estágio de três semanas.

No segundo momento, após o treinamento de três semanas com o guia, os estudantes foram avaliados com a mesma ficha aplicada no primeiro dia.

Em relação às informações sobre os achados de exame físico, as variáveis foram categóricas, com três possibilidades (exame completo, exame parcial e exame ausente). Assim, cada uma dessas possibilidades foi tratada como uma variável dicotômica. A avaliação da efetividade da intervenção educacional, para a melhoria da qualidade do preenchimento de aspectos da avaliação clínica, foi feita por meio da comparação entre os dois conjuntos de dados obtidos antes e após a intervenção, para cada item de avaliação clínica; para tanto,aplicou-se o teste estatístico de McNemar, para amostras pareadas.

Além disso, foram obtidos os escores globais de qualidade da avaliação por meio do somatório de pontos atribuídos a cada item de avaliação clínica analisado (1,0 ponto para "completo"; 0,5 ponto para "parcial"; zero ponto para "ausente"). Este somatório foi dividido por 1,3. Assim, cada aluno tinha um escore global mínimo de zero ponto e um máximo de dez pontos, sendo, portanto, considerada uma variável contínua. Foram comparados os escores globais antes e depois do treinamento; para isto, primeiro, aplicou-se o teste de Kolmogorov-Smirnov para verificar a normalidade da variável. Caso a distribuição encontrada fosse normal, aplicar-se-ia o teste $t$ pareado; já se fosse uma distribuição não normal, seria aplicado o teste de Wilcoxon.

Adicionalmente, foi avaliada a possível associação entre o ano cursado de graduação de Medicina e a pontuação do escore global obtida após o treinamento. Tal análise foi feita pelo teste de correlação de Pearson. Para todas as análises, foi assumido $\mathrm{p}<0,05$.

\section{RESULTADOS}

Ao todo, foram avaliados 31 estudantes, sendo que 19 examinaram pacientes do sexo feminino e 12 examinaram pacientes do sexo masculino.

No período que precedeu o treinamento dos alunos, os itens mais avaliados foram o sistema cardiovascular (100\%), sistema pulmonar $(100 \%)$, abdome $(100 \%)$ e sinais vitais $(83,87 \%)$, seguidos por palpação de linfonodos $(35,48 \%)$, exame neurológico $(25,81 \%)$, palpação da tireoide $(16,13 \%)$, exame da cavidade oral (12,90\%), medidas antropométricas $(9,68 \%)$ e mamas (5,26\%). De outro modo, a fundoscopia, a otoscopia, o exame dos testículos e a avaliação do ITB foram ignorados por todos os alunos (Gráfico 1). Após o treinamento, os mais avaliados, por ordem decrescente, foram o sistema cardiovascular (100\%), sistema pulmonar (100\%), abdome $(100 \%)$, sinais vitais $(100 \%)$, palpação de linfonodos $(100 \%)$, exame da cavidade oral (96,77\%), exame neurológico (93,55\%), índice tornozelo-braquial (90,32\%), otoscopia (74,19\%), medidas antropométricas $(70,97 \%)$, mamas $(68,42 \%)$, palpação da tireoide $(67,74 \%)$, fundoscopia $(35,48 \%)$ e, finalmente, testícu$\operatorname{los}(25 \%)$ (Gráfico 2). 


\section{GrÁfico 1}

Frequência relativa dos itens de avaliação clínica atendidos pelos alunos, de modo parcial ou completo, antes do treinamento $(\mathbf{N}=31)$

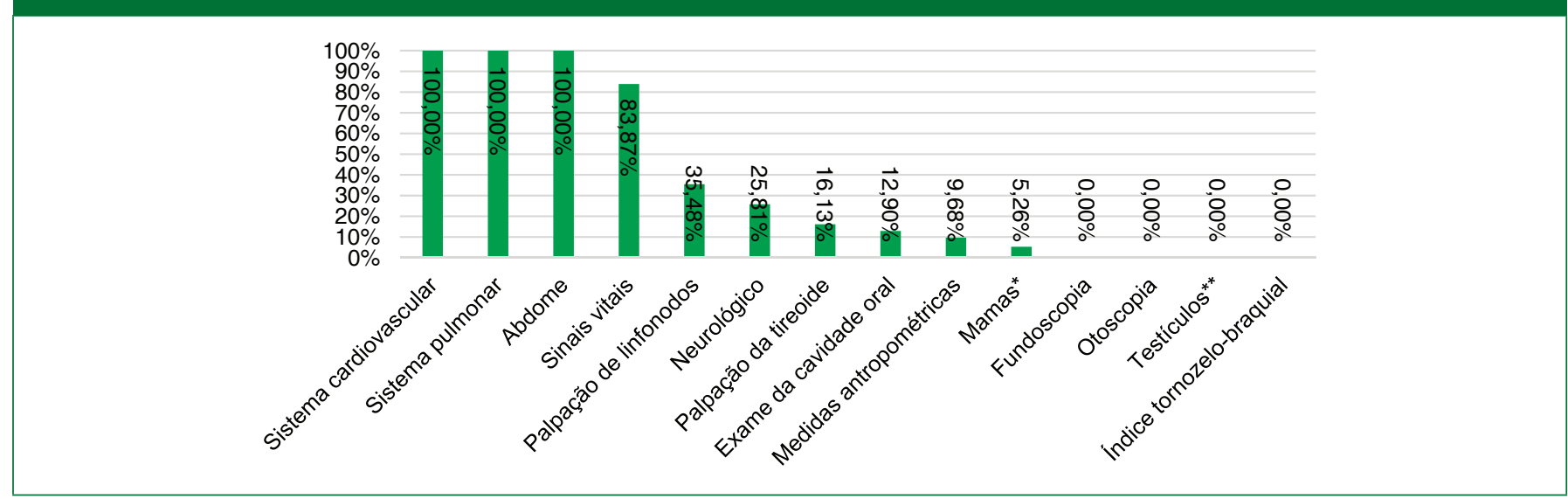

Fonte: Dados coletados do estudo $(\mathrm{CHJB} / 2014) .{ }^{*} \mathrm{~N}=19 .{ }^{* *} \mathrm{~N}=12$.

\section{GrÁfico 2}

Frequência relativa dos itens de avaliação clínica atendidos pelos alunos, de modo parcial ou completo, depois do treinamento $(\mathbf{N}=31)$

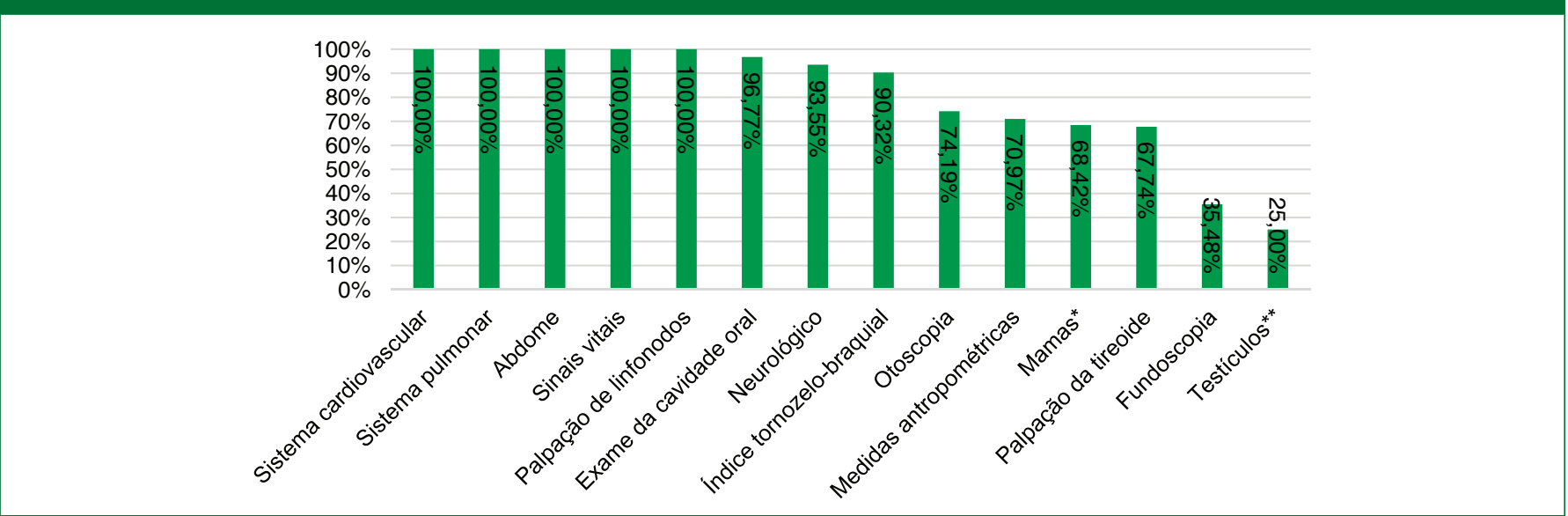

Fonte: Dados coletados do estudo (CHJB/2014). ${ }^{*} N=19 .{ }^{* *} N=12$.

O percentual de alunos que realizaram avaliação completa dos sinais vitais aumentou de modo significativo, de $16,13 \%$, antes do treinamento, para $100 \%$, após o treinamento, com risco relativo $(\mathrm{RR})$ de $6,2(\mathrm{P}<0,01)$. Tal aumento da qualidade da avaliação resultou na significativa e desejada ausência de avaliações consideradas parciais $(\mathrm{P}<0,01)$ e ausentes $(\mathrm{P}$ $=0,06)$, conforme observado na Tabela 2 .

Resultados desejados e estatisticamente significativos foram observados em relação à análise das avaliações clínicas completas, do sistema cardiovascular (3,23\% versus $74,19 \%$; $\mathrm{RR}=23 ; \mathrm{P}<0,01)$, pulmonar $(22,58 \%$ versus $90,32 \%$; $\mathrm{RR}=4$;
$\mathrm{P}<0,01)$, abdome (22,58\% versus $74,19 \%$; $\mathrm{RR}=3.29 ; \mathrm{P}=0,01)$, neurológico ( $0 \%$ versus $22,58 \%$; RR = não estimável; $\mathrm{P}=0,02)$, dos linfonodos $(6,45 \%$ versus $77,42 \%$; $R R=12 ; P<0,01)$, tireoide $(0 \%$ versus $61,29 \%$; RR = não estimável; $\mathrm{P}<0,01)$, cavidade oral $(6,45 \%$ versus $67,74 \%$; $R R=10,5 ; \mathrm{P}<0,01)$, fundoscopia $(0 \%$ versus $32,26 \%$; $R R=$ não estimável; $\mathrm{P}<0,01)$, otoscopia $(0 \%$ versus $64,52 \% ; \mathrm{RR}=$ não estimável; $\mathrm{P}<0,01)$, mamas $(0 \%$ versus $36,84 \%$; RR = não estimável; $\mathrm{P}=0,02)$, medidas antropométricas ( $0 \%$ versus $45,16 \%$; RR = não estimável; $\mathrm{P}<0,01)$ e índice tornozelo-braquial ( $0 \%$ versus $83,87 \%$; RR = não estimável; $\mathrm{P}<0,01)$. 
TABELA 1

Frequência relativa dos itens de avaliação clínica atendidos pelos alunos, de modo parcial ou completo, antes e depois do treinamento

\begin{tabular}{lcc} 
Domínio semiológico & $\begin{array}{c}\text { Antes } \\
(\mathbf{N}=\mathbf{3 1}) \\
\mathbf{\%}\end{array}$ & $\begin{array}{c}\text { Depois } \\
(\mathbf{N = 3 1 )} \\
\%\end{array}$ \\
\hline Sistema cardiovascular & 100 & 100 \\
Sistema pulmonar & 100 & 100 \\
\hline Abdome & 100 & 100 \\
Sinais vitais & 83,87 & 100 \\
Palpação de linfonodos & 35,48 & 100 \\
\hline Neurológico & 25,81 & 93,55 \\
Palpação da tireoide & 16,13 & 67,74 \\
Exame da cavidade oral & 12,90 & 96,77 \\
\hline Medidas antropométricas & 9,68 & 70,97 \\
Mamas* & 5,26 & 68,42 \\
Fundoscopia & 0 & 35,48 \\
Otoscopia & 0 & 74,19 \\
Testículos** & 0 & 25 \\
Índice tornozelo-braquial & 0 & 90,32
\end{tabular}

Fonte: Dados coletados do estudo (CHJB/2014). ${ }^{*} N=19 .{ }^{* *} N=12$.

TABELA 2

Comparações dos percentuais de avaliações dos domínios semiológicos considerados completos, parciais e ausentes, antes e depois do treinamento $(\mathrm{N}=31)$

\begin{tabular}{llrrrr} 
Domínios avaliado & Qualidade & $\begin{array}{c}\text { \% Antes } \\
(\mathbf{N}=31)\end{array}$ & $\begin{array}{c}\text { \% Depois } \\
(\mathbf{N}=31)\end{array}$ & RR & \multicolumn{1}{c}{ P } \\
Sinais vitais & Completo & 16,13 & 100,00 & 6,20 & $<0,01$ \\
& Parcial & 67,74 & 0,00 & 42,00 & $<0,01$ \\
& Ausente & 16,13 & 0,00 & 10,00 & 0,06 \\
Palpação de & Completo & 6,45 & 77,42 & 12,00 & $<0,01$ \\
linfonodos & Parcial & 29,03 & 22,58 & 0,78 & 0,79 \\
& Ausente & 64,52 & 0,00 & 40,00 & $<0,01$ \\
Palpação da & Completo & 0,00 & 61,29 & $\mathrm{NE}$ & $<0,01$ \\
tireoide & Parcial & 16,13 & 6,45 & 0,40 & 0,45 \\
& Ausente & 83,87 & 32,26 & 0,38 & $<0,01$ \\
\multirow{4}{*}{ Sistema } & Completo & 3,23 & 74,19 & 23,00 & $<0,01$ \\
cardiovascular & Parcial & 96,77 & 25,81 & 0,27 & $<0,01$ \\
& Ausente & 0,00 & 0,00 & $\mathrm{NE}$ & 1,00 \\
Sistema & Completo & 22,58 & 90,32 & 4,00 & $<0,01$ \\
pulmonar & Parcial & 77,42 & 9,68 & 0,13 & $<0,01$ \\
& Ausente & 0,00 & 0,00 & $\mathrm{NE}$ & 1,00 \\
& Completo & 22,58 & 74,19 & 3,29 & 0,01 \\
Abdome & Parcial & 77,42 & 25,81 & 0,33 & $<0,01$ \\
& Ausente & 0,00 & 0,00 & $\mathrm{NE}$ & 1,00 \\
& Completo & 0,00 & 22,58 & $\mathrm{NE}$ & 0,02 \\
Neurológico & Parcial & 25,81 & 70,97 & 2,75 & $<0,01$ \\
& Ausente & 74,19 & 6,45 & 0,09 & $<0,01$
\end{tabular}

TABELA 2

Comparações dos percentuais de avaliações dos domínios semiológicos considerados completos, parciais e ausentes, antes e depois do treinamento $(\mathbf{N}=31)$

\begin{tabular}{|c|c|c|c|c|c|}
\hline Domínios avaliados & s Qualidade & $\begin{array}{l}\% \text { Antes } \\
(\mathrm{N}=31)\end{array}$ & $\begin{array}{c}\text { \% Depois } \\
(\mathrm{N}=31)\end{array}$ & RR & $\mathbf{P}$ \\
\hline \multirow{3}{*}{$\begin{array}{l}\text { Exame da } \\
\text { cavidade oral }\end{array}$} & Completo & 6,45 & 67,74 & 10,50 & $<0,01$ \\
\hline & Parcial & 6,45 & 29,03 & 4,50 & 0,07 \\
\hline & Ausente & 87,10 & 3,23 & 0,04 & $<0,01$ \\
\hline \multirow{3}{*}{ Fundoscopia } & Completo & 0,00 & 32,26 & $\mathrm{NE}$ & $<0,01$ \\
\hline & Parcial & 0,00 & 3,23 & $\mathrm{NE}$ & 1,00 \\
\hline & Ausente & 100,00 & 64,52 & 0,65 & $<0,01$ \\
\hline \multirow{3}{*}{ Otoscopia } & Completo & 0,00 & 64,52 & $\mathrm{NE}$ & $<0,01$ \\
\hline & Parcial & 0,00 & 9,68 & $\mathrm{NE}$ & 0,25 \\
\hline & Ausente & 100,00 & $25,81^{* * *}$ & 0,26 & $<0,01$ \\
\hline \multirow{3}{*}{ Mamas* } & Completo & 0,00 & 36,84 & $\mathrm{NE}$ & 0,02 \\
\hline & Parcial & 5,26 & 31,58 & 6,00 & 0,13 \\
\hline & Ausente & 94,74 & $31,58^{* * *}$ & 0,33 & $<0,01$ \\
\hline \multirow{3}{*}{ Testículos** } & Completo & 0,00 & 8,33 & $\mathrm{NE}$ & 1,00 \\
\hline & Parcial & 0,00 & 16,67 & $\mathrm{NE}$ & 0,50 \\
\hline & Ausente & 100,00 & 75,00 & 0,75 & 0,25 \\
\hline \multirow{3}{*}{$\begin{array}{l}\text { Medidas } \\
\text { antropométricas }\end{array}$} & Completo & 0,00 & 45,16 & $\mathrm{NE}$ & $<0,01$ \\
\hline & Parcial & 9,68 & 25,81 & 2,67 & 0,22 \\
\hline & Ausente & 90,32 & $29,03^{* * *}$ & 0,32 & $<0,01$ \\
\hline \multirow{3}{*}{$\begin{array}{l}\text { Índice tornozelo- } \\
\text { braquial }\end{array}$} & Completo & 0,00 & 83,87 & $\mathrm{NE}$ & $<0,01$ \\
\hline & Parcial & 0,00 & 6,45 & $\mathrm{NE}$ & 0,50 \\
\hline & Ausente & 100,00 & 9,68 & 0,10 & $<0,01$ \\
\hline
\end{tabular}

Fonte: Dados coletados do estudo $(\mathrm{CHJB} / 2014) .{ }^{*} N=19 .{ }^{* *} N=12 .{ }^{* * *}$ Dos casos de "avaliação ausente", um foi considerado ausente devido à indisponibilidade de informação na ficha de avaliação. Valores de P obtidos por meio do teste estatístico de McNemar. RR: Risco relativo. NE: Não estimável.

A única exceção das comparações de avaliações completas foi observada nos exames dos testículos, com ausência total de avaliações completas antes do treinamento dos alunos e aumento discreto para 8,33\%, após o treinamento. Tal diferença foi considerada insignificante, segundo análise estatística $(\mathrm{P}=1,0)$.

A comparação dos desempenhos globais individuais antes (mediana $=1,92$; valor máximo: 5,38, valor mínimo: 1,15 ) e após (mediana $=7,31$; valor máximo: 10,0; valor mínimo: $4,23)$ do período de treinamento demonstrou uma diferença estatística significativa ( $\mathrm{p}<0,01$ ), conforme observado na Tabela 3 .

O aumento no desempenho, segundo as pontuações globais nas avaliações clínicas individuais, demonstrou fraca correlação ( $\mathrm{R} 2=0,1242 ; \mathrm{p}=0,0515)$ com o ano cursado de Medicina, conforme demonstrado pelo Gráfico 3. 
TABELA 3

Comparação das medianas dos desempenhos globais individuais entre os dois períodos de avaliação, antes e após o período de treinamento

$\begin{array}{cccc}\begin{array}{c}\text { Pontuação global } \\ \text { antes (mediana, } \\ \text { mín. e máx.) } \\ \mathbf{N}=31\end{array} & \begin{array}{c}\text { Pontuação global } \\ \text { após (mediana, } \\ \text { mín. e máx.) } \\ \mathbf{N}=31\end{array} & \begin{array}{c}\text { Diferença entre } \\ \text { medianas }\end{array} & \mathbf{P} \\ 1,92(1,15-5,38) & 7,31(4,23-10,0) & 5,38 & <0,01\end{array}$

Fonte: Dados coletados do estudo $(\mathrm{CHJB/2014)}$. Mín. $=$ valor mínimo. Máx $=$ valor máximo. Valores de P obtidos por meio do teste estatístico de Wilcoxon.

\section{DISCUSSÃO}

A falta de adesão à prática de avaliações clínicas ou exames físicos adequados constitui uma realidade mundial que vem sendo constatada nas últimas décadas ${ }^{13,14}$. Este problema é tão presente e bem relatado nos serviços de ensino médico de todo o mundo, que um dos autores desta área, Chad Cook, especialista em doenças musculoesqueléticas, refere o exame clínico como "A arte perdida"15.

O presente estudo demonstra a baixa valorização do exame físico antes do treinamento (Tabelas 1 e 2). Embora alguns segmentos do exame clínico tenham sido registrados em $100 \%$ dos casos (exame cardiovascular, pulmonar e abdominal), muitos prontuários demonstraram estar incompletos, segundo a Tabela 2.
Este cenário encontrado antes do treinamento é semelhante aos resultados de outros estudos. Um estudo multicêntrico realizado por Barrios et al. ${ }^{16}$, em 1995, por exemplo, demonstrou que a realização do exame físico já era negligenciada em grandes centros universitários mesmo que os pacientes apresentassem queixas que justificassem o exame. Dez anos mais tarde, os mesmos autores analisaram os exames físicos realizados por doutorandos e residentes no Serviço de Medicina Interna de um hospital universitário e observaram que o sistema cardiovascular, o sistema pulmonar e o abdome eram privilegiados em relação aos demais, registrados respectivamente, nas seguintes porcentagens: 94,1\%, 95,8\% e 91,66\%. Entretanto, apesar deste registro, raramente ele foi realizado de forma completa. Este dado é exemplificado nos casos do sistema pulmonar (completo em apenas 10\%) e do aparelho cardiovascular $(46,6 \%)$. Além disso, da totalidade dos pacientes, apenas $11,6 \%$ dos casos apresentaram descrição do exame neurológico nas notas de internação (14/120 pacientes) ${ }^{13}$. Estes dados são semelhantes aos do presente estudo, em que, segundo a Tabela 1, o sistema cardiovascular, o sistema pulmonar e o abdome foram registrados em $100 \%$ dos pacientes antes do treinamento com o guia; porém, a Tabela 2 mostra que apenas 3,23\%, 22,58\% e $22,58 \%$ dos pacientes tiveram avaliações consideradas completas para o sistema cardiovascular, o pulmonar e o abdome, respectivamente. Além disso, a avaliação neurológica foi realizada em $25,81 \%$ dos pacientes, mas todas ainda de forma parcial, antes do treinamento com o guia.

Gráfico 3

Correlação entre o ano cursado de Medicina e os desempenhos globais individuais na avaliação clínica, após o treinamento

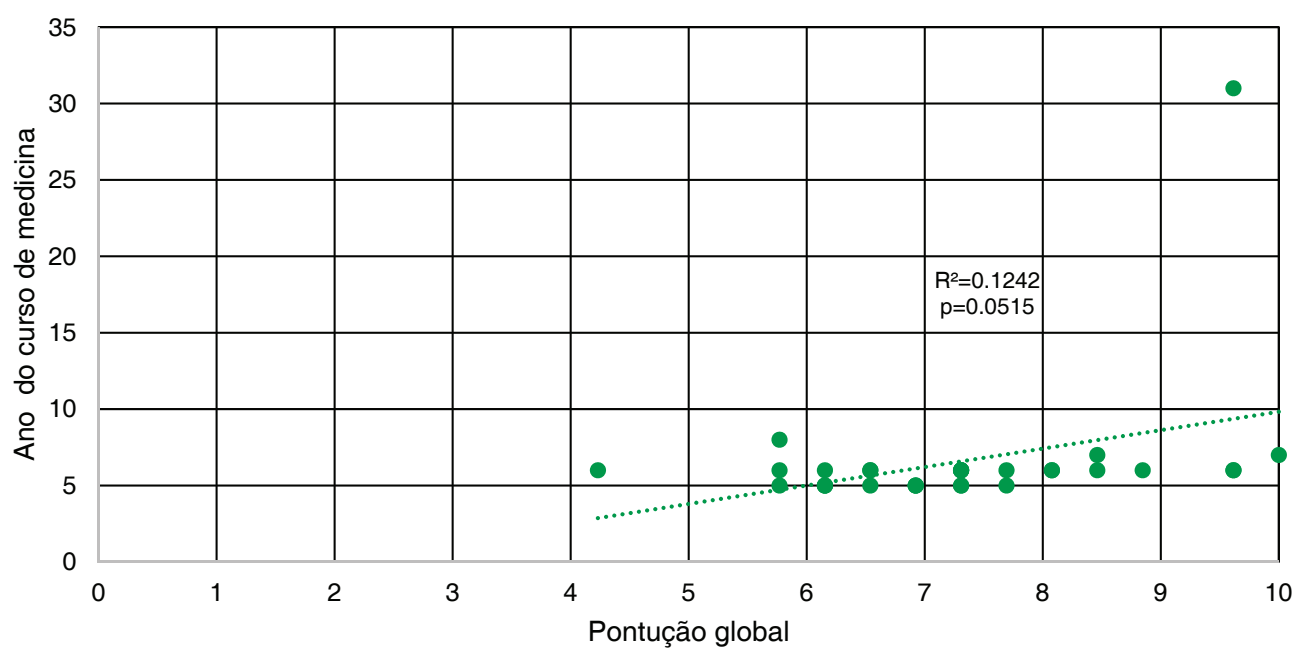

Fonte: Dados coletados do estudo (CHJB/2014). Valor de P obtido por meio do teste estatístico de Pearson. 
Em outro trabalho realizado, os autores utilizaram um escore clínico construído com base em dados de exame físico por ocasião do atendimento médico e conforme informações do paciente. Tal escore clínico foi construído com base em questões dirigidas ao paciente que diziam respeito aos seguintes procedimentos médicos: (1) aferição da tensão arterial; (2) ausculta cardíaca; (3) ausculta pulmonar; (4) palpação do abdome; (5) após mostrar o estetoscópio e o tensiômetro, se tais aparelhos foram usados durante o exame físico. Desse modo, o escore clínico poderia variar de 0 a 5 pontos, sendo o escore zero atribuído à consulta médica sem aparente investigação clínica, e o 5 àquela na qual o exame físico foi supostamente mais adequado. O escore clínico registrou média de 3,1 ( $\pm 2,1)$, sendo $23,9 \%$ (34/142) com o escore zero. Dos 142 pacientes incluídos neste estudo, $50(35,2 \%)$ referiram que a tensão arterial não foi aferida, também 50 (35,2\%) não tiveram o tórax auscultado e de quase a metade $(47,2 \% ; n=67)$ o abdome não foi examinado ${ }^{17}$.

Ainda corroborando os resultados do presente estudo, Silva e Rezende ${ }^{18}$, em 2008, observaram que 65\% dos alunos de Medicina, que já tinham passado pela semiologia médica de adultos, se autoavaliaram sem a habilidade necessária para realizar um exame físico, e 18,5\% referiram não perceber sinais semiológicos importantes.

$\mathrm{O}$ exame neurológico pode ter tido um baixo percentual de avaliações completas (22,58\%) mesmo após o treinamento (Tabela 2) devido à "neurofobia", que é um fenômeno em que os estudantes de Medicina muitas vezes percebem a neurologia como a especialidade médica mais difícil, o que faz com que não queiram lidar com condições neurológicas ${ }^{19}$. A complexidade do exame clínico é um dos principais motivos para muitos estudantes acharem a neurologia difícil ${ }^{20}$. Os estudos mostram que os alunos de Medicina geralmente apresentam desempenho mais fraco no exame neurológico do que em outros campos de avaliação clínica ${ }^{21}$.

A palpação dos linfonodos melhorou substancialmente: $100 \%$ dos alunos avaliaram os linfonodos após o treinamento, sendo $77,42 \%$ de forma completa. Provavelmente, o guia simplificado do estudo, que lista as cadeias ganglionares a serem avaliadas pelos estudantes em cada segmento corporal, auxiliou de maneira significativa nessa melhora, pois, de forma simples e sequencial, os alunos poderiam lembrar de quais cadeias deveriam ser examinadas.

A fundoscopia apresentou melhora sensível após a intervenção. Porém, apenas 35,48\% dos alunos realizaram o exame, de modo completo ou parcial (Gráfico 2), mesmo tendo um oftalmoscópio à disposição. Isto demonstra a grande dificuldade de ensino da oftalmoscopia direta na graduação. A literatura médica já constatou que a maioria dos clínicos e es- tudantes não apresenta a confiança e a habilidade necessárias para realizar uma oftalmoscopia ${ }^{22}$. Um estudo evidenciou que os estudantes, para realizar um exame oftalmológico, tinham um nível de confiança médio abaixo de 2, numa escala de 1 a 5 (1 sendo o menos confiante $)^{23}$. Então, deve haver uma mudança nesse contexto, pois é necessário que o clínico saiba como realizar uma oftalmoscopia direta e como interpretá-la. Para tanto, é necessário que haja mais exposição dos alunos a consultórios de oftalmologia ${ }^{22}$. Um estudo criou um oftalmoscópio de ensino, em que uma terceira pessoa poderia observar a visão que o estudante estava tendo do fundo do olho durante o exame ${ }^{24}$. Outra opção com menor custo seria ter mais sessões específicas de treinamento de habilidades para o exame oftalmológico, utilizando-se materiais menos onerosos ${ }^{23}$.

A otoscopia teve melhora significativa após a intervenção, chegando a $64,52 \%$ de avaliação completa (Tabela 2). Antes do treinamento com o guia, nenhum estudante realizou a otoscopia, o que demonstra que o ensino da realização da otoscopia é muito deficiente durante a graduação também. Jones et al. ${ }^{25}$, por exemplo, identificaram que apenas $5 \%$ dos estudantes de Medicina avaliados sentiam que poderiam realizar um exame adequado de otoscopia em crianças. Outro estudo mostrou que apenas 38,5\% dos médicos de atenção primária do San Antonio Military Health System utilizam otoscópio para diagnóstico de otite média aguda ${ }^{26}$.

O exame da tireoide passou de $0 \%$ para $61,29 \%$ de avaliações completas. A avaliação clínica desta glândula é pouco realizada na prática médica, tanto que Wu et al. ${ }^{27}$, em 2007, encontraram um nível de autoconfiança de 2,8 (escala de 1 a 5, sendo 1 o menor nível de confiança) entre os estudantes de Medicina na detecção de um nódulo na tireoide. Porém, com simples intervenções, este cenário é passível de mudança, como foi visto no presente estudo e no trabalho de Haring et al. ${ }^{28}$, em 2014. Esses autores notaram que, após um treinamento sistemático de habilidades clínicas e a realização do exame físico em pacientes reais, 75\% dos alunos de Medicina realizaram o exame de tireoide. E 75\% destes realizaram o exame de forma adequada.

Em relação ao exame das mamas, percebe-se que apenas $5,26 \%$ dos estudantes o realizaram, e ainda de forma parcial, antes da intervenção com o guia. Após o treinamento, houve um aumento para $68,42 \%$, porém ainda houve um baixo percentual de avaliações consideradas completas (36,84\%). Isto provavelmente se deve a barreiras culturais por se tratar de uma área íntima da mulher. Wang et al. ${ }^{29}$, em 2015, demonstraram, numa pesquisa feita por meio de um questionário, que a aceitabilidade de o exame das mamas ser realizado por médicos homens é baixa entre os estudantes de Medicina e os próprios médicos, com $35,03 \%$ e $59,66 \%$ de aceitabilidade, respectivamente. Haring et al. ${ }^{28}$, apresentaram dados mais preocupantes ainda em 
relação ao exame de mamas: nesse estudo, cem estudantes de Medicina passaram por oito semanas de treinamento sistemático de habilidades clínicas, seguidas por mais oito semanas de treinamento em pacientes reais. No final, observou-se que apenas nove alunos realizaram a palpação das mamas.

A avaliação dos testículos foi o único item que não foi associado a qualquer diferença educacional e estatística considerada relevante, provavelmente por ocasião de regras culturais, percepção de baixa distribuição de doenças relacionadas na população geral e também provavelmente associado ao descaso dos alunos para com a importância da prática deste tipo de avaliação na fase atual de suas formações. Para superar essa barreira da avaliação clínica genital, talvez sejam necessárias medidas mais profundas, como um programa de ensino integrado sobre o exame genital feminino-masculino (utilizando professores treinados e pacientes simulados em um ambiente de ensino clínico supervisionado), pois, durante a graduação, existem poucas oportunidades de praticar esse tipo de exame ${ }^{30}$.

O exame da cavidade oral foi realizado em 96,77\% dos pacientes após o treinamento, um dado concordante com o estudo de Haring et al. ${ }^{28}$, de 2014, que demonstrou que $100 \%$ dos alunos realizaram o exame da cavidade oral e da faringe após uma intervenção educacional. Porém, Haring et al..$^{28}$ notaram que apenas $9 \%$ desses alunos realizaram o exame de maneira adequada. Já no presente estudo, 67,74\% das avaliações foram consideradas completas (Tabela 2). Essa discrepância pode ter ocorrido devido às diferenças nos critérios para se considerar este exame adequado.

$\mathrm{O}$ índice tornozelo-braquial (ITB) passou de 0\% para $83,87 \%$ de avaliação completa. A deficiência prévia no conhecimento acerca do ITB entre os médicos já foi mostrada em outros estudos. No estudo de Wyatt et al. ${ }^{31}$, em 2010, foi demonstrado que, entre 29 residentes de medicina interna, apenas $4 \%$ mensuraram de forma correta o ITB, 10\% calcularam corretamente e $45 \%$ interpretaram corretamente o ITB. Porém, após uma sessão educacional com um especialista, houve uma melhora sensível: $50 \%$ dos residentes mensuraram de forma correta, $75 \%$ calcularam corretamente e $88 \%$ interpretaram de forma adequada. Então, uma intervenção simples, como o guia simplificado, pode melhorar consideravelmente a mensuração do ITB.

As medidas antropométricas melhoraram após o treinamento. Porém, a avaliação completa ficou em 45,16\%, percentual relativamente baixo, considerando-se que o IMC e a circunferência abdominal são simples de serem feitos. Talvez a percepção, por parte dos estudantes, da pouca utilidade dessas medidas durante a internação e o deslocamento do paciente do seu leito a uma balança para se medir altura e peso tenham sido fatores que prejudicaram a avaliação deste item.
O Gráfico 3 demonstrou não haver correlação entre o tempo já cursado de graduação do estudante e a pontuação global após a intervenção. Isto pode ter ocorrido devido à maior parte dos alunos ter diferença de apenas um ano de curso (quinto ano e sexto ano de Medicina); logo, essa diferença de tempo pode não ser tão significativa para a realização do exame físico. Ademais, o pequeno número de participantes no estudo, 31 no total, pode ter influenciado.

Para solucionar o atual problema no processo de formação médica, que não prioriza a habilidade de realizar um exame clínico completo na rotina, a preparação e a conscientização dos professores sobre esta competência na graduação são a principal estratégia recomendada ${ }^{32,33}$. Além disso, os autores do presente estudo sugerem que o treinamento com o guia simplificado para doutorandos e residentes no início do estágio na presença de um professor seja outra estratégia de simples execução e provavelmente muito eficaz. Estabelecer um programa integrado com a disciplina de Semiologia de acompanhamento dos alunos até o final do curso poderia ser uma iniciativa de fácil implementação. Mas é válido reforçar a necessidade de testar, além das instruções presenciais e materiais impressos, outros recursos, como os eletrônicos, que podem incluir a internet e aplicativos ${ }^{34,35}$.

Ressaltando a eficiência do guia simplificado de exame físico, os alunos do presente estudo aperfeiçoaram claramente a semiotécnica clínica, com um aumento de 280,7\% na pontuação mediana do desempenho dos alunos após o treinamento (1,92 versus 7,31 pontos), segundo a Tabela 3. Neste sentido, uma sequência padrão de exame físico que contempla todos os sistemas demonstrou ser efetiva para a quase totalidade dos itens de avaliação clínica analisados.

O ensino de habilidades clínicas, especialmente o de avaliações clínicas, exames físicos ou outras situações da prática dos cuidados em saúde, pode ser influenciado por diferentes estilos de ensino, diferenças nas equipes de professores de uma instituição, características administrativas locais, grau de individualização e características do paciente e do avaliador ${ }^{36,37}$. Apesar de tais influências, além de certos fatores subjetivos, instrumentos objetivos, em formato de checklist, são extremamente promissores e recomendáveis para os próximos estudos nesta área, como meio viável para o processo de ensino-aprendizagem de avaliações clínicas ${ }^{38}$.

Além da utilização na formação de alunos de graduação em Medicina, o instrumento proposto poderia também ser utilizado em ações de educação médica continuada em vários serviços de saúde, tornando-os presumivelmente mais eficientes e responsáveis sobre a utilização de tecnologias diagnósticas, principalmente em países de baixa renda ${ }^{39}$. 
É interessante notar que, no presente estudo, os alunos exerceram as atividades de modo autônomo, sem a presença de um professor, no momento exato das avaliações ${ }^{40}$. Diferentemente dos Exames Clínicos Estruturados e Objetivos, o guia simplificado de avaliação clínica foi aplicado em circunstâncias compatíveis com as demandas reais do serviço de saúde no qual o estudo foi conduzido (Complexo Hospitalar Jean Bitar). Desde 1975, a metodologia dos Exames Clínicos Estruturados e Objetivos incorpora uma ampla variedade de atividades em casos, cenários e situações muito diferentes, mas todos em caráter de simulação, inclusive com a participação de atores, quando necessário ${ }^{41}$. Este aspecto foi enfatizado por Weller et al. 2 , em 2014, no artigo intitulado Can I leave the theatre? A key to more reliable workplace-based assessment (Posso abandonar o teatro? A chave para avaliações confiáveis baseadas em ambientes de trabalho). Nesse estudo, os autores enfatizam que métodos de avaliação em situações de maior independência dos alunos, em situações reais, podem constituir meios confiáveis de avaliação, sem onerar a rotina de ambos, ensino e serviço.

É evidente que há a limitação de o guia simplificado ter sido aplicado durante apenas três semanas. Para uma análise mais aprimorada e uma intervenção mais profunda, seria necessário um tempo maior. Mas este estudo já demonstra a efetividade dessa ferramenta e serve como base para estudos futuros. Além disso, outras intervenções podem ser necessárias juntamente com o guia simplificado, principalmente nos itens que ainda tiveram uma avaliação completa relativamente baixa.

\section{CONCLUSÃO}

Há um grande déficit no ensino de habilidades clínicas durante a graduação de Medicina. Como uma solução eficaz, um guia simplificado sequencial de exame clínico pode servir de treinamento a estudantes de Medicina.

\section{REFERÊNCIAS}

1. Störmann S, Stankiewicz M, Raes P, Berchtold C, Kosanke $Y$, Illes $G$, et al. How well do final year undergraduate medical students master practical clinical skills? GMS J Med Educ 2016; 33(4): Doc58.

2. Mangione S, Nieman LZ, Gracely E, Kaye D. The teaching and practice of cardiac auscultation during internal medicine and cardiology training. A nationwide survey. Ann Intern Med 1993; 119(1):47-54.

3. Mavis BE. Does studying for an objective structured clinical examination make a difference? Med Educ 2000; 34(10): 808-12.

4. Lacombe MA. On bedside teaching. Ann Intern Med 1997; 126(3):217-20.

5. Thibault GE. Bedside rounds revisited. N Engl J Med 1997; 336(16):1174-5.
6. Obel J. Losing the touch: as technology and medical education change, doctors May Lose the ability to perform physical exams. The Washington Post. 2003 jun 17, HE01.

7. Holmboe ES. Faculty and the observation of trainees' clinical skills: problems and opportunities. Acad Med 2004; 79(1):16-22.

8. Epstein RM, Hundert EM. Defining and assessing professional competence. JAMA 2002; 287(2):226-35.

9. Gordon J, Hazlett C, Ten Cate O, Mann K, Kilminster S, Prince $\mathrm{K}$, et al. Strategic planning in medical education: enhancing the learning environment for students in clinical settings. MedEduc 2000; 34(10):841-50.

10. Associação brasileira para o Estudo da Obesidade e da Síndrome Metabólica. Diretrizes brasileiras de obesidade 2016 [accessed 05 december 2016]. Disponível em: http:/ / www. abeso.org.br/uploads/downloads/92/57fccc 403e5da.pdf

11. GiolloJúnior LT, Martin JFV. Índice tornozelo-braquial no diagnóstico da doença aterosclerótica carotídea. RevBrasHipertens 2010; 17(2):117-8.

12. Nunes FGF, Leão GCS, Exel AL, Diniz MCC. Índice tornozelo-braquial em pacientes de alto risco cardiovascular. RevBrasCardiol 2012;25(2):94-101.

13. Barrios CH, Silva VL, Pinheiro RF, Oliveira RL, Gasnier R. Avaliação do registro do exame físico realizado por doutorandos e residentes no Serviço de Medicina Interna do Hospital São Lucas: PUCRS. Sci Med 2005; 15(3):156-61.

14. Feddock CA. The lost art of clinical skills. Am J Med 2007; 120(4): 374-8

15. Cook C. The lost art of the clinical examination: an overemphasis on clinical special tests. J Man ManipTher 2010;18(1):3-4.

16. Barrios $\mathrm{CH}$, Fogliatto L, Mota LM. O rei está nu: o exame físico em três hospitais universitários de Porto Alegre. Rev AMRIGS 1995; 39:123-7.

17. Ney-oliveira F, Neto AMS, Santos MB, Tavares-Neto J. Relação entre a qualidade do exame clínico e o acerto na requisição da radiografia de tórax. RadiolBras 2005;38(3):187-93.

18. Silva RMFL, Rezende NA. O ensino de semiologia médica sob a visão dos alunos: implicações para a reforma curricular. Rev Bras Educ Med 2008; 32(1):32-9.

19. Abushouk AI, Duc NM. Curing neurophobia in medical schools: evidence-based strategies. Med Educ Online 2016; 21:32476.

20. Matthias AT, Nagasingha P, Ranasinghe P, Gunatilake SB. Neurophobia among medical students and non-specialist doctors in Sri Lanka. BMC MedEduc 2013; 13:164.

21. Roze E, Flamand-Roze C, Meneret A, Ruiz M, Le Liepvre $\mathrm{H}$, Duguet A, et al. 'The Move', an innovative simulation- 
-based medical education program using roleplay to teach neurological semiology: Students' and teachers' perceptions. RevNeurol (Paris) 2016; 172(4-5):289-94.

22. Yusuf IH, Salmon JF, Patel CK. Direct ophthalmoscopy should be taught to undergraduate medical students-yes. Eye (Lond) 2015; 29(8):987-9.

23. Hoonpongsimanont W, Nguyen K, Deng W, Nasi, D, Chakravarthy B, Lotfipour S. Effectiveness of a 40-minute Ophthalmologic Examination Teaching Session on Medical Student Learning. West J Emerg Med 2015; 16(5):721-6.

24. Schulz C, Moore J, Hassan D, Tamsett E, Smith CF. Addressing the 'forgotten art of fundoscopy': evaluation of a novel teaching ophthalmoscope. Eye (Lond) 2016; 30(3):375-84.

25. Jones WS, Johnson C, Longacre J. How well are we teaching otoscopy? Medical students perspectives. Pediatr Res 2003; 53 suppl:95A.

26. Harvey M, Bowe SN, Laury AM. Clinical Practice Guidelines: Whose Practice Are We Guiding? Otolaryngol Head Neck Surg 2016; 155(3):373-5.

27. Wu EH, Fagan MJ, Reinert SE, Diaz JA. Self-confidence in and perceived utility of the physical examination: a comparison of medical students, residents, and faculty internists. J Gen Intern Med 2007; 22(12):1725-30.

28. Haring CM, Cools BM, Van der Meer JW, Postma CT. Student performance of the general physical examination in internal medicine: an observational study. BMC Med Educ 2014;14:73.

29. Wang YJ, Yang J, Kang LX, Jia Z, Chen DM, Zhang P, et al. Acceptability of physical examination by maledoctors in medical care: Taking breast palpation as an example. J HuazhongUnivSciTechnolog Med Sci 2015; 35(5):781-4.

30. McBain L, Pullon S, Garrett S, Hoare K. Genital examination training: assessing the effectiveness of an integrated female and male teaching programme. BMC Med Educ 2016; 16(1):299.

31. Wyatt MF, Stickrath C, Shah A, Smart A, Hunt J, Casserly IP. Ankle-brachial index performance among internal medicine residentes. Vasc Med 2010; 15(2):99-105.

32. Schattner A. Revitalizing the history and clinical examination. Am J Med 2012; 125(4):e1-3.

33. Breckwoldt J, Svensson J, Lingemann C, Gruber H. Does clinical teacher training always improve teaching effectiveness as opposed to no teacher training? A randomized controlled study. BMC Med Educ 2014;14:6.

34. Azer AS, Algrain HA, Alkhelaif RA, Aleshaiwi SM. Evaluation of the educational value of YouTube videos about physical examination of the cardiovascular and respiratory systems. J Med Internet Res 2013;15(11):e241.
35. Sondhi V, Devgan A. Translating technology into patient care: Smartphone applications in pediatric health care. Med J Armed Forces India 2013; 69(2):156-61.

36. Lawry GV 2nd, Schuldt SS, Kreiter CD, Densen P, Albanese MA. Teaching a screening musculoskeletal examination: a randomized, controlled trial of different instructional methods. Acad Med 1999; 74(2):199-201.

37. Longworth MK. An exploration of the perceived factors that affect the learning and transfer of skills taught to student midwives. Midwifery 2013; 29(8): 831-7.

38. Conigliaro RL, Stratton TD. Assessing the quality of clinical teaching: a preliminary study. Med Educ 2010; 44(4):379-86.

39. Ethiopia. University of Gondar. Physical diagnosis: lecture notes for health science students. [accessed 06 december 2016]. Disponívelem: https://www.cartercenter.org/resources/pdfs/health/ephti/library/lecture_notes/health_science_students/ln_phys_diagnosis_final.pdf

40. Swaffield S. Getting to the heart of authentic Assessment for Learning. Assess Educ 2011; 18(4):433-49.

41. Tormey W. Education, learning and assessment: current trends and best practice for medical educators. Ir J Med Sci 2014;184(1):1-12.

42. Weller JM, Misur M, Nicolson S, Morris J, Ure S, Crossley J, et al. Can I leave the theatre? A key to more reliable workplace-based assessment. Br J Anaesth 2014;112(6):1083-91.

\section{CONTRIBUIÇÃO DOS AUTORES}

Salomão Neto - idealização do trabalho e do guia simplificado, interpretação dos resultados. Tiago Braga: pesquisa bibliográfica, edição dos dados e elaboração do artigo. Márcia Portella - orientação, edição e coordenação da pesquisa. Regis Andriolo - orientação, edição e coordenação da pesquisa.

\section{CONFLITO DE INTERESSES}

Declaramos que não há conflito de interesses entre os autores deste artigo.

\section{ENDEREÇO PARA CORRESPONDÊNCIA}

Salomão Neto

Centro Hospitalar Jean Bitar

Rua Cônego Jerônimo Pimentel, 543

Umarizal - Belém

CEP 66055-000 - PA

E-mail: matheus98@oi.com.br 